\title{
Produktportfolio RHI Magnesita - Erweiterung durch MgO-basierte Produkte im Bereich Pulp \& Paper
}

\author{
Sonja Larissegger ${ }^{1}$, Erich Feichtenhofer ${ }^{1}$ und Helmut Flachberger ${ }^{2}$ \\ ${ }^{1} \mathrm{RHI}$ Magnesita GmbH, Leoben, Österreich \\ ${ }^{2}$ Lehrstuhl für Aufbereitung und Veredlung, Department Mineral Resources Engineering, Montanuniversität \\ Leoben, Leoben, Österreich
}

Eingegangen 5. Juli 2019; angenommen 10. Juli 2019; online publiziert 7. August 2019

\begin{abstract}
Zusammenfassung: Für einen erfolgreichen Einsatz von synthetischer kaustischer Magnesia (Kauster) von RHI Magnesita (RHIM) in der Papier- und Zellstoffindustrie muss der ofenfallende und damit zu grob vorliegende Kauster einem Zerkleinerungsschritt unterzogen werden. Zur Auffindung der dafür am besten geeigneten Zerkleinerungsmethode und deren Einflüsse auf zwei prozessbestimmende Parameter, das Sedimentationsverhalten und den erreichbaren Hydratisierungsgrad, wurden bei unterschiedlichen Maschinenherstellern Zerkleinerungsprodukte hergestellt und auf deren spezifische Eigenschaften untersucht.

Der als "RHIM CCM Ofenfallend" bezeichnete Ausgangsrohstoff, welcher auf Basis des Meerwasserprozesses hergestellt wird, stammt aus dem in Norwegen situierten Werk Porsgrunn von RHIM. Vom „RHIM CCM Ofenfallend” wurden mit Hilfe verschiedener Zerkleinerungsaggregate zerkleinerte MgO-Proben hergestellt, mittels Dispersitätsanalysen charakterisiert (z. B. erzielte doo-Werte und BET-Oberflächen) und schließlich im Labormaßstab hinsichtlich des Sedimentationsverhaltens und des Hydratisierungsgrades analysiert. Die mit Hilfe einer Stiftmühle hergestellte MgOProbe zeigte dabei die besten Ergebnisse, daher wurde dieser Mühlentyp für die weitere Aufbereitung einer Großmenge an „RHIM CCM Ofenfallend“ herangezogen. Das daraus resultierende, gemahlene Kausterprodukt „RHIM CCM $P \& P^{\prime \prime}$ wurde in einem Betriebsversuch bei einem namhaften Zellstoffproduzenten eingehend getestet. Das Kausterprodukt "RHIM CCM P\&P“ konnte erfolgreich eingesetzt und die nach Auswertung des Betriebsversuches erkannten Vorteile aufgezeigt werden.
\end{abstract}

Schlüsselwörter: Synthetischer Kauster, Magnesiumoxid,

\footnotetext{
H. Flachberger ( $\square)$

Lehrstuhl für Aufbereitung und Veredlung, Department Mineral

Resources Engineering,

Montanuniversität Leoben,

Leoben, Österreich

helmut.flachberger@unileoben.ac.at
}

Hydratisierung, Magnesiumhydroxid, Pulp \& Paper, Zellstoffproduktion, Zerkleinerungsaggregat, Hydratisierungsgrad, Sedimentationsverhalten

Product Portfolio RHI Magnesita-Add-on with MgObased Products for Pulp \& Paper

Abstract: Successful application of synthetic caustic calcined magnesia (CCM) in the pulp \& paper industry needs previous investigation of processing methods and their influence on the sedimentation behaviour and reachable degree of hydration. The basic raw material, produced by the sea water process, comes from RHI Magnesita, the global market leader in the refractory industry, from the plant in Norway.

RHIM CCM was treated with different crushing aggregates to produce crushed $\mathrm{MgO}$-samples. These samples were tested in lab scale regarding sedimentation behaviour and degree of hydration. The best results were shown by the sample which was prepared with the pin mill, therefore the pin mill was selected to treat a larger amount of RHIM CCM. "RHIM CCM P\&P" was the product of this process route and was tested in a large-scale trial at a production plant of a well-known pulp producer. The "RHIM CCM P\&P" was successfully used, and after the evaluation of the trial, the advantages of the application of this product can be demonstrated.

Keywords: Synthetic caustic calcined magnesia, Magnesium oxide, Hydration, Magnesium hydroxide, Pulp \& Paper, Pulp production, Crushers, Degree of hydration, Sedimentation behaviour

\section{Einleitung}

RHI Magnesita (RHIM), weltweit größter Hersteller von Feuerfestprodukten, geht neue Wege. Angesichts des herausfordernden Umfeldes in einem globalen MgO-Roh- 
stoffmarkt werden neue Möglichkeiten gesucht, um die Marktführerschaft des Unternehmens auch in Zukunft zu sichern. Die Diversifikation im Bereich des Rohstoffverkaufes gewinnt dadurch stark an Bedeutung. Hier konzentriert sich RHIM auf Kundensegmente, die nicht dem Feuerfestbereich zuzuordnen sind, dazu gehören zum Beispiel die Futtermittel- oder Schweiß-Industrie. Das Produktportfolio des Rohstoffverkaufes wird dafür kontinuierlich über das Kerngeschäft hinaus mit MgO-basierenden Produkten für unterschiedlichste Kundenapplikationen erweitert. Die neueste Entwicklung ist der Einsatz von synthetischer Magnesia im Bereich Pulp \& Paper im Prozessschritt der Zellstoffproduktion.

Um den Eintritt in ein neues Marktsegment positiv zu bewältigen, müssen zwei Grundvoraussetzungen gegeben sein, nämlich die ökonomischen Voraussetzungen und die Rohstoffverfügbarkeit. Die ökonomischen Voraussetzungen sind dadurch gegeben, dass die Welternährungsorganisation (FAO) 2010 die Prognose abgab, der weltweite Papierbedarf werde bis 2050 jährlich um ca. $3 \%$ steigen [1]. Der wachsende Bedarf an Papier und Karton wird durch die in Abb. 1 ausgewiesene Marktstatistik der Confederation of European Paper Industry (CEPI) bestätigt [2]. Dieser Aufwärtstrend wird zusätzlich noch durch die aktuelle Diskussion über das sogenannte "Mikroplastik" und den zunehmenden Onlinehandel, welcher einen starken Anstieg im Verbrauch an Verpackungsmaterial zur Folge hat, verstärkt.

Der für die Anwendung in Pulp \& Paper geeignete Rohstoff wird von RHI Magnesita in Norwegen am Standort Porsgrunn produziert. Das auf synthetischem MgO basierende Rohgut wird über die Meerwasserprozessroute hergestellt. Dabei wird gebrannter Dolomit aufgeschlämmt und mit Meerwasser vermischt. Das somit gewonnene $\mathrm{Mg}(\mathrm{OH})_{2}$ wird anschließend gewaschen, entwässert und durch Brennen in einem Drehrohrofen bei ca. $800^{\circ} \mathrm{C} z \mathrm{zu}$ kaustischer Magnesia umgewandelt. Ein Großteil davon wird in Elektrolichtbogenöfen weiter zu Schmelzmagnesia verarbeitet. Der restliche Produktstrom wird aufbereitet, um die physikalischen Eigenschaften für unterschiedlichs-

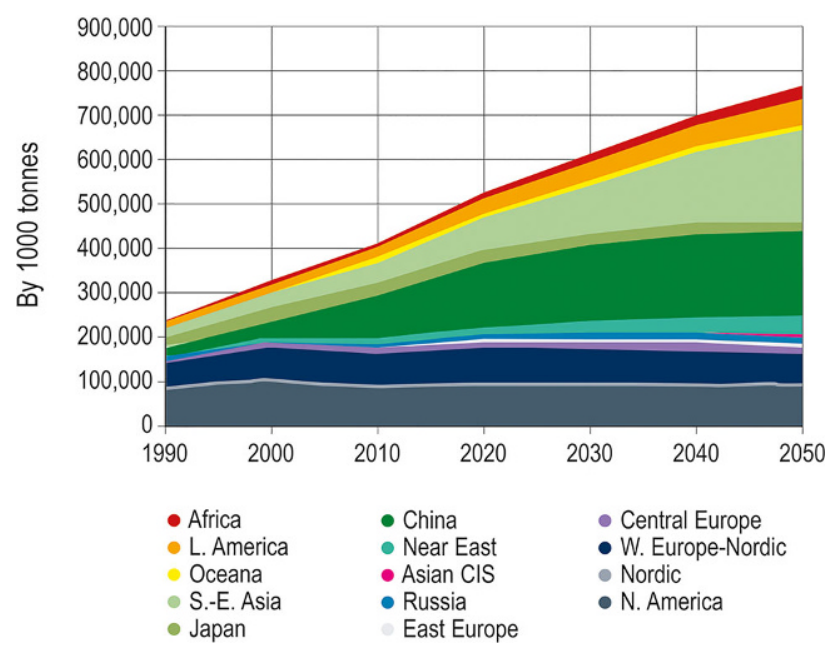

Abb. 1: Langfristige Entwicklung des Papier-und Kartonbedarfes nach Regionen bis 2050 [2]

\begin{tabular}{|c|c|c|}
\hline Kriterium & Einheit & Wert \\
\hline $\mathrm{MgO}$ & $\%$ & 94,9 \\
\hline $\mathrm{CaO}$ & $\%$ & 1,65 \\
\hline $\mathrm{SiO}_{2}$ & $\%$ & 0,25 \\
\hline $\mathrm{Fe}_{2} \mathrm{O}_{3}$ & $\%$ & 0,20 \\
\hline $\mathrm{Al}_{2} \mathrm{O}_{3}$ & $\%$ & 0,05 \\
\hline $\mathrm{MnO}$ & $\%$ & 0,02 \\
\hline Glühverlust & $\%$ & 2,5 \\
\hline
\end{tabular}

te Anwendungen optimal einzustellen. Durch die Verwendung von Meerwasser und qualitativ hochreinem Dolomit wird synthetischer Kauster höchster Qualität erhalten. Die chemische Zusammensetzung unterliegt keinen Schwankungen, da immer dieselben hochwertigen Ausgangsmaterialien verwendet werden. Die chemische Zusammensetzung des synthetischen Kausters "RHIM CCM Ofenfallend“ ist in Tab. 1 zusammengefasst.

\section{Verwendung von Magnesia in der Zellstoffproduktion}

In der Zellstoffproduktion wird MgO bevorzugt im sauren Bisulfitverfahren, dass zu den chemischen Aufschlussverfahren von Holz gehört, eingesetzt. Dabei wird der Zellstoff durch Kochen von Holzchips mit Magnesiumbisulfitlösung im sauren Milieu, der sogenannten Kochsäure, unter erhöhter Temperatur und/oder erhöhtem Druck gewonnen. Nach dem Kochprozess wird die Ablauge, die nach Extraktion der Zellulose zurückbleibt, verbrannt. Das Bisulfitverfahren wird bevorzugt eingesetzt, weil das $\mathrm{MgO}$ und das $\mathrm{SO}_{2}$, welches bei der Verbrennung der Ablauge (Bezeichnung für die mit organischen Substanzen aus dem Holz angereicherte Kochsäure) entsteht, rückgewonnen werden können. Bei Verwendung von Calcium-basierenden Basenbestandteilen ist keine Rückgewinnung möglich, da bei der Verbrennung der Calciumbisulfit-Ablauge schwerlöslicher Gips $\left(\mathrm{CaSO}_{4} \cdot 2 \mathrm{H}_{2} \mathrm{O}\right)$ entsteht [2].

\subsection{Rückgewinnung von $\mathrm{MgO}$ und $\mathrm{SO}_{2}-$ Herstellung der Kochsäure}

Die aus der Verbrennung der Ablauge rückgewonnenen $\mathrm{SO}_{2}$ - und $\mathrm{MgO}-$ Anteile werden zur Herstellung der Kochsäure verwendet. Das $\mathrm{MgO}$ wird durch Elektrofilter aus dem Rauchgas abgeschieden und dient wieder zur Rauchgasentschwefelung des bei der Verbrennung der Ablauge produzierten Rauchgases. Hierzu wird das $\mathrm{MgO}$ zu Magnesiumhydroxid hydratisiert und anschließend mit dem Rauchgas in Kontakt gebracht. Die Absorption des $\mathrm{SO}_{2}$ wird durch das in Lösung vorliegende $\mathrm{Mg}(\mathrm{OH})_{2}$ in mehrstufigen Venturikaskaden bewerkstelligt (siehe GI. 1-4). Durch die Absorption 
von $\mathrm{SO}_{2}$ an $\mathrm{Mg}(\mathrm{OH})_{2}$ entsteht dann das Magnesiumbisulfit (Kochsäure) [3, 4].

$$
\begin{aligned}
& \mathrm{MgO}+\mathrm{H}_{2} \mathrm{O} \leftrightarrow \mathrm{Mg}(\mathrm{OH})_{2} \\
& \mathrm{Mg}(\mathrm{OH})_{2}+\mathrm{SO}_{2} \leftrightarrow \mathrm{MgSO}_{3}+\mathrm{H}_{2} \mathrm{O} \\
& \mathrm{MgSO}_{3}+\mathrm{H}_{2} \mathrm{O}+\mathrm{SO}_{2} \leftrightarrow \mathrm{Mg}\left(\mathrm{HSO}_{3}\right)_{2} \\
& \text { Gesamt : } \mathrm{MgSO}_{3}+2 \mathrm{SO}_{2} \leftrightarrow \mathrm{Mg}\left(\mathrm{HSO}_{3}\right)_{2}
\end{aligned}
$$

Nachdem das $\mathrm{MgO}$ mit einer Quote von ca. $90 \%$ rezykliert wird und die Verluste durch Zugabe von frischem MgO (sogenanntes Make-Up MgO) ausgeglichen werden, muss das $\mathrm{MgO}$ möglichst hochwertig sein und geringe Störstoffgehalte aufweisen. In der Zellstoffindustrie gehören zu den Störstoffen Kalk ( $\mathrm{CaO})$, Siliciumdioxid $\left(\mathrm{SiO}_{2}\right)$, Eisen(III)-oxid $\left(\mathrm{Fe}_{2} \mathrm{O}_{3}\right)$ und Manganoxid ( $\left.\mathrm{MnO}\right)$. CaO kann mit dem im System vorhandenen $\mathrm{SO}_{2}$ zu erheblichen Gipsablagerungen führen. $\mathrm{SiO}_{2}$ wird als Begleitstoff des $\mathrm{MgO}$ betrachtet und hat zwar keinen negativen Einfluss auf den chemischen Prozess, erhöht aber den Verschleiß an den Anlagenkomponenten, insbesondere bei den Pumpen und Rohrleitungen. $\mathrm{Fe}_{2} \mathrm{O}_{3}, \mathrm{Al}_{2} \mathrm{O}_{3}$ und $\mathrm{MnO}$ wirken sich nach der Kochung in der Zellstoffbleiche negativ auf den Chemikalienverbrauch im Bleichprozess aus [3].

\subsection{Hydratisierung von $\mathrm{MgO} z u \mathrm{Mg}(\mathrm{OH})_{2}$}

Ein besonderes Augenmerk gilt dem ersten Schritt, der Umsetzung von $\mathrm{MgO}$ zu $\mathrm{Mg}(\mathrm{OH})_{2}$ durch Hydratisierung. Die Reaktion läuft nach der theoretischen GI. 5 ab und ist mit $+38 \mathrm{~kJ} / \mathrm{mol}$ leicht exotherm. Für $\mathrm{MgO}$ mit einer spezifischen Oberfläche von $20-40 \mathrm{~m}^{2} / \mathrm{g}$, das dadurch ausreichend Reaktivität aufweist, kann die Hydrierung bei atmosphärischem Druck durchgeführt werden [5].

$$
\mathrm{MgO}+\mathrm{H}_{2} \mathrm{O} \rightarrow \mathrm{Mg}(\mathrm{OH})_{2}+\text { Wärme }
$$

Voraussetzung für eine gleichmäßige Umsetzung von $\mathrm{MgO} \mathrm{zu} \mathrm{Mg}(\mathrm{OH})_{2}$ ist eine homogen vorhandene Aktivität im MgO. Durch die Homogenität des Ausgangsmaterials können Posthydratisierungseffekte, wie zum Beispiel die Erhöhung der Viskosität der Lösung, vermieden werden [5].

Bei näherer Betrachtung der Reaktion aus GI. 5 besteht die Hydratisierung von $\mathrm{MgO}$ zu $\mathrm{Mg}(\mathrm{OH})_{2}$ aus einer Auflöseund Abscheidereaktion. Zuallererst wird durch das vorliegende Wasser die MgO-Oberfläche protoniert und dadurch positiv geladen (GI. 6). Die Anwesenheit dieser positiven Ladungen ermöglicht die Dissoziation des Zwischenproduktes $\mathrm{MgOH}^{+}$(GI. 7). Die niedrige Löslichkeit von $\mathrm{Mg}(\mathrm{OH})_{2}$ im $\mathrm{pH}$-Bereich von neutral bis stark basisch begünstigt die Abscheidung von $\mathrm{Mg}(\mathrm{OH})_{2}$ möglichst nahe an der $\mathrm{MgO}$ Oberfläche (GI. 8). Vor allem die Eigenschaften des Reaktionsmediums (Temperatur und Feststoffgehalt) sowie die MgO-Quelle (Monokristalle oder polykristalline Körnung) beeinflussen die Schritte, die in den GIn. 6-8 dargestellt

\begin{tabular}{|c|c|c|}
\hline \multicolumn{3}{|c|}{$\begin{array}{l}\text { TABELLE } 2 \\
\text { Festgelegte Basis-Qualitätskriterien für MgO zum } \\
\text { Einsatz in der Zellstoffproduktion }\end{array}$} \\
\hline Kriterium & Einheit & Wert \\
\hline $\mathrm{MgO}$ & $\%$ & Min. 90 \\
\hline $\mathrm{CaO}$ & $\%$ & Max. 2,2 \\
\hline $\mathrm{SiO}_{2}$ & $\%$ & Max. 2,5 \\
\hline $\mathrm{Fe}_{2} \mathrm{O}_{3}$ & $\%$ & Max. 0,8 \\
\hline $\mathrm{MnO}$ & $\%$ & Max. 0,4 \\
\hline Glühverlust & $\%$ & Max. 5,0 \\
\hline $\mathrm{d}_{90}$ & $\mu \mathrm{m}$ & $<100$ \\
\hline Hydratisierungsgrad & $\%$ & $>60$ \\
\hline
\end{tabular}
sind $[6,7]$.

$$
\begin{aligned}
& \mathrm{MgO}+\mathrm{H}_{2} \mathrm{O} \rightarrow \mathrm{MgOH}^{+}+\mathrm{OH}^{-} \\
& \mathrm{MgOH}^{+}+\mathrm{OH}^{-} \rightarrow \mathrm{Mg}^{2+}+2 \mathrm{OH}^{-}
\end{aligned}
$$

$$
\mathrm{Mg}^{2+}+2 \mathrm{OH}^{-} \rightarrow \mathrm{Mg}(\mathrm{OH})_{2}
$$

In der Praxis unterscheiden sich die Temperaturbedingungen und Verweilzeiten bei der Hydratisierung von $\mathrm{MgO}$ zu $\mathrm{Mg}(\mathrm{OH})_{2}$ stark. Der Temperaturbereich erstreckt sich von $30-90^{\circ} \mathrm{C}$, die Verweilzeiten liegen zwischen $15-75 \mathrm{~min}$, wobei manche Zellstoffhersteller die Hydratisierung in mehreren Stufen mit einem ansteigenden Temperaturprofil durchführen, andere hingegen führen hierfür nur einen einstufigen Prozess durch.

\section{Definition der Basis-Qualitätskriterien für Kaustische Magnesia zum Einsatz in der Zellstoffproduktion}

Gemeinsam mit ausgewählten Zellstoffproduzenten wurden die wichtigsten chemischen und physikalischen BasisQualitätskriterien des einzusetzenden MgO erarbeitet (siehe Tab. 2). Dabei wurde besonderes Augenmerk auf das Sedimentationsverhalten und den Hydratisierungsgrad (d. h. die Umsetzung von $\mathrm{MgO}$ zu $\mathrm{Mg}(\mathrm{OH})_{2}$ ) gelegt.

\subsection{Sedimentationsverhalten}

Eine wichtige Eigenschaft ist das Sedimentationsverhalten des Feststoffes in der hergestellten $\mathrm{Mg}(\mathrm{OH})_{2}$-Suspension. Diese muss pumpfähig sein und die Feststoffe sollen sich nicht in den Anlagenteilen absetzen. Vor allem bei Ausfall von Rühr- und Pumpvorrichtungen ist dies ein wichtiger Punkt, da eine zu rasche und kompakte Sedimentation wichtige Anlagenteile blockieren kann (wie zum Beispiel die Rührvorrichtungen im Hydratisierungsbehälter). Daher wurden die Korngrößenverteilungen des ofenfallenden MgO-Produktes von RHIM und einigen am Markt erhältlichen Konkurrenzprodukten analysiert und eine obere Korngrößengrenze bei einem $\mathrm{d}_{90}<100 \mu \mathrm{m}$ eruiert und als zu erreichende Zielgröße festgelegt, um zum einen das Erreichen hoher Basis-Qualitätskriterien sicherzustellen bzw. um zum anderen keine Ablagerungen bei Einsatz im großindustriellen Maßstab zu verursachen und eine gute Mischbarkeit von $\mathrm{MgO}$ mit Wasser zu erzielen. 


\subsection{Hydratisierungsgrad}

Der Hydratisierungsgrad stellt ein Maß für die Umsetzung von $\mathrm{MgO}$ zu $\mathrm{Mg}(\mathrm{OH})_{2}$ dar und ist somit die wichtigste Eigenschaft. Dabei sind vor allem die Temperatur und der Feststoffgehalt der Suspension sowie die MgO-Quelle (Monokristalle oder polykristalline Körnung) von Bedeutung [7].

Je größer der Anteil an vorhandenem $\mathrm{Mg}(\mathrm{OH})_{2}$ in der Suspension, umso besser kann das bei der Verbrennung der Dicklauge produzierte Rauchgas entschwefelt werden. In weiterer Folge kann dadurch ausreichend Magnesiumbisulfit zum Kochen der Holzchips produziert werden.

\section{Experimenteller Teil}

Der vorliegende "RHIM CCM Ofenfallend“ weist eine Korngrößenverteilung von 0-2 mm direkt aus dem Drehrohrofen auf. Um den Korngrößenbereich mit einem d 90 von $<100 \mu \mathrm{m}$ zu erhalten, wurde der "RHIM CCM Ofenfallend“ mittels unterschiedlicher Aufbereitungsaggregate gemahlen. Die hierfür verwendeten Aggregate waren: eine Walzenschüsselmühle, eine Stiftmühle und eine Mahlanlage, deren Zerkleinerungswirkung auf der Ausnutzung von Schallstoßwellen beruht.

Die dabei erhaltenen zerkleinerten $\mathrm{MgO}$-Proben wurden einer Korngrößenbestimmung unterzogen und anschlieBend für die weiterführenden Versuche zur Analyse des Sedimentationsverhaltens und die Experimente zur Bestimmung des Hydratisierungsgrades verwendet.

\subsection{Analyse des Sedimentationsverhaltens}

Die zerkleinerten MgO-Proben wurden $1 \mathrm{~h}$ bei konstanter, vorab festgelegter Feststoffkonzentration (90 und $70 \mathrm{~g} / \mathrm{l}$ ) und eingestellter Temperatur mit Hilfe eines Magnetrührers in Wasser gerührt. Danach wurde die $\mathrm{Mg}(\mathrm{OH})_{2}$-Suspension in eine Mensur überführt und die Sedimentation des $\mathrm{Mg}(\mathrm{OH})_{2}$ für die Dauer von $1 \mathrm{~h}$ beobachtet. Zu Beginn der Sedimentation wurde nach 5, 10 und $15 \mathrm{~min}$ die rasch entstehende Grenzfläche zwischen Klarwasserschicht und $\mathrm{Mg}(\mathrm{OH})_{2}$-Suspension (siehe Abb. 2) notiert, danach jeweils in Abständen von $15 \mathrm{~min}$.

Das nach einer bestimmten Zeit erreichte $\mathrm{Mg}(\mathrm{OH})_{2}$-Volumen wird mit dem Startvolumen bei 0 min (entspricht $100 \%$ ) ins Verhältnis gesetzt und in Prozent vom Startvolumen angegeben. Im Bereich des linearen Sedimentationsverhaltens wird die Sedimentationsgeschwindigkeit nach GI. 9 berechnet. Der zurückgelegte Weg s entspricht dabei dem durch die Sedimentationsgrenzfläche zurückgelegten Weg vom Startvolumen bis zum Zeitpunkt $x$.

$$
v_{\text {Sed }}=\frac{s}{t} \quad \text { in } \mathrm{m} / \mathrm{s}
$$

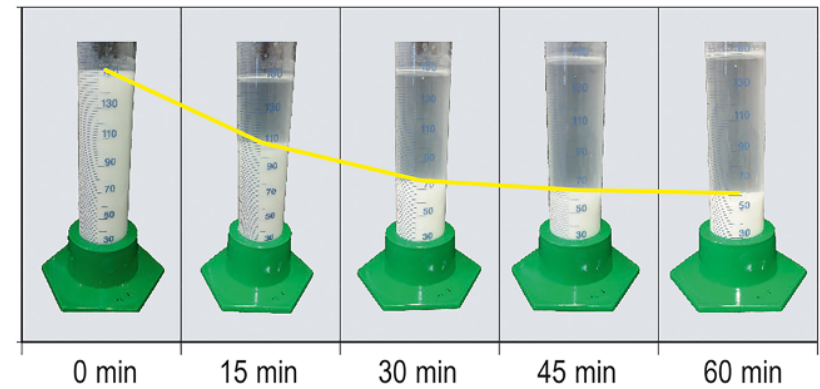

Abb. 2: Schematische Darstellung des Sedimentationsverhaltens

\subsection{Bestimmung des Hydratisierungsgrades}

Die in Abschn. 4.1 hergestellte Suspension wurde geteilt, ein Teil wurde für die Beobachtung der Sedimentation verwendet, der zweite Teil der Suspension wurde abfiltriert, mit Ethanol nachgewaschen, um die Hydratisierungsreaktion zu stoppen, und der Filterkuchen anschließend im Trockenschrank bis zur Gewichtskonstanz bei $105^{\circ} \mathrm{C}$ getrocknet. Danach wurde die getrocknete Probe analysefein gemahlen und mittels Thermogravimetrischer Analyse (TGA) vermessen. Zum Vergleich wurde eine MgO-Probe (Originalprobe), die nicht mit Wasser behandelt wurde, ebenfalls analysefein gemahlen und mit TGA vermessen, diese Daten dienen als Basis für die folgende Berechnung des zu erreichenden Hydratisierungsgrades.

Daraus ergibt sich für die Originalprobe der Gehalt an Wasser (GI. 10), die aufgenommene Luftfeuchtigkeit, und der Gehalt an MgO (GI. 11). Der Gehalt an Wasser wird in Mol-Wasser umgerechnet (GI. 12) und auf die Einwaage pro g bezogen (GI. 13).

$$
\begin{aligned}
& m_{\mathrm{H}_{2} \mathrm{O}}=\frac{m_{E W} \cdot \% \text { LOI }}{(-100)} \\
& m_{\text {Original }}=m_{E W}-m_{\mathrm{H}_{2} \mathrm{O}} \\
& n_{\mathrm{H}_{2} \mathrm{O}}=\frac{m_{\mathrm{H}_{2} \mathrm{O}}}{M\left(\mathrm{H}_{2} \mathrm{O}\right)} \\
& N_{\mathrm{H}_{2} \mathrm{O} \text { Original }}=\frac{n_{\mathrm{H}_{2} \mathrm{O}}}{m_{E W}}
\end{aligned}
$$

Die hydratisierten Proben werden anschließend ausgewertet. Dazu erfolgt die Berechnung wie für die Originalprobe nach GIn. 10-12. Danach wird der enthaltene Wasseranteil der Originalprobe abgezogen (Gln. 14 und 15).

$$
\begin{aligned}
& n_{\mathrm{H}_{2} \mathrm{O} \text { Original }}=N_{\mathrm{H}_{2} \mathrm{O} \text { Original }} * m_{E W \text { Probe }} \\
& n_{\mathrm{H}_{2} \mathrm{O} \text { Probe }}=n_{\mathrm{H}_{2} \mathrm{O}}-n_{\mathrm{H}_{2} \mathrm{O} \text { Original }}
\end{aligned}
$$

Weiters wird der Anteil an MgO in der zu vermessenden Probe mit Hilfe der Röntgenfluoreszenz-Analyse (RFA) bestimmt und berechnet (GIn. 16 und 17).

$$
\begin{aligned}
& m_{\mathrm{MgO}}=m_{\text {Probe }} * \frac{\%_{\mathrm{MgO} \text { RFA }}}{100} \\
& n_{\mathrm{MgO}}=\frac{m_{\mathrm{MgO}}}{M(\mathrm{MgO})}
\end{aligned}
$$




\begin{tabular}{|c|c|c|c|c|c|}
\hline \multicolumn{6}{|c|}{$\begin{array}{l}\text { TABELLE } 3 \\
\text { Ermittelte Korngrößenkennwerte } d_{10}, d_{50} \text { und } d_{90} \text { an } \\
\text { den zerkleinerten MgO-Proben (nach Aufbereitung } \\
\text { von „RHIM CCM Ofenfallend“' mit unterschiedlichen } \\
\text { Zerkleinerungsaggregaten }\end{array}$} \\
\hline $\begin{array}{l}\text { Krite- } \\
\text { rium }\end{array}$ & $\begin{array}{l}\text { Ein- } \\
\text { heit }\end{array}$ & $\begin{array}{l}\text { RHIM } \\
\text { CCM Ofen- } \\
\text { fallend }\end{array}$ & $\begin{array}{l}\text { Stift- } \\
\text { mühle }\end{array}$ & $\begin{array}{l}\text { Walzen- } \\
\text { schüssel- } \\
\text { mühle }\end{array}$ & $\begin{array}{l}\text { Schall- } \\
\text { stoß- } \\
\text { wellen }\end{array}$ \\
\hline$d_{10}$ & $\mu \mathrm{m}$ & 1100 & 1 & 3 & 0,1 \\
\hline $\mathrm{d}_{50}$ & $\mu \mathrm{m}$ & 650 & 2 & 15 & 2 \\
\hline $\mathrm{d}_{90}$ & $\mu \mathrm{m}$ & 300 & 8 & 104 & 8 \\
\hline
\end{tabular}

Die enthaltene tatsächliche Menge an Wasser wird in Verhältnis zur vorhandenen Menge an MgO gesetzt und damit der Hydratisierungsgrad in \% erhalten (GI. 18).

$$
H G=\frac{n_{\mathrm{H}_{2} \mathrm{O} \text { Probe }} * 100}{n_{\mathrm{MgO}}}
$$

\section{Ergbnisse}

Im folgenden Kapitel werden die Ergebnisse im Detail für die Sedimentation und den zu erreichenden Hydratisierungsgrad dargestellt und interpretiert.

\subsection{Sedimentationsverhalten}

Da die Sedimentation durch die Korngröße beeinflusst wird, wurden von allen zerkleinerten MgO-Proben ergänzend die Korngrößenverteilungen bestimmt.

\subsubsection{Bestimmung der Korngrößenverteilung}

Die Analyse der Korngrößenverteilung wurde mittels Laserbeugung (in Ethanol mit Ultraschall) oder trocken mit Hilfe

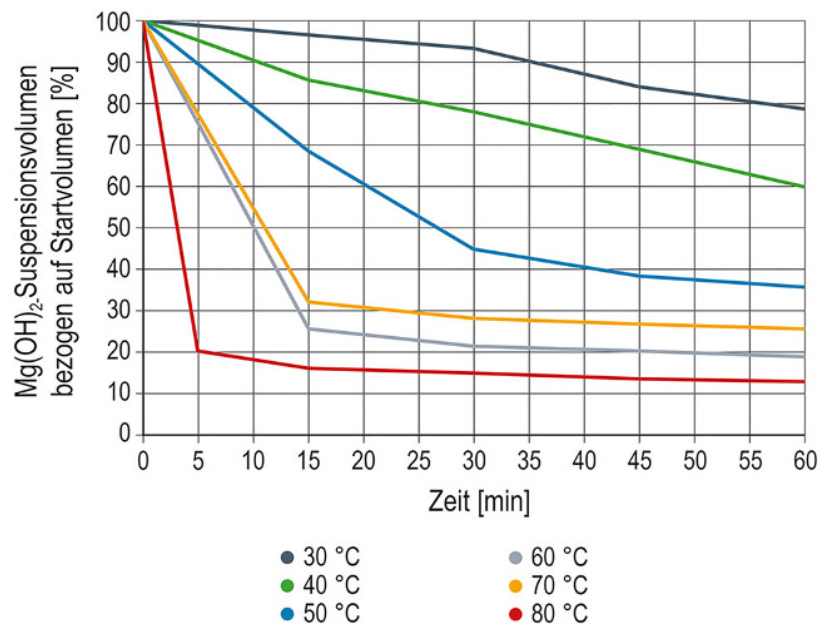

Abb. 3: Sedimentationsverhalten „RHIM CCM Ofenfallend“ nach unterschiedlichen Hydratisierungstemperaturen (Hydratisierungsdauer $1 \mathrm{~h}$ ) mit einer Konzentration von $90 \mathrm{~g} / \mathrm{l}$ von Siebmaschinen durchgeführt. Die Ergebnisse sind in Tab. 3 zusammengefasst.

Durch den Vergleich in Tab. 3 ist zu erkennen, dass der "RHIM CCM Ofenfallend“ gut mit den verwendeten Aggregaten zerkleinert werden konnte. Die zerkleinerten MgOProben von Stiftmühle und Schallstoßwellen weisen $\mathrm{d}_{90}$ Werte von jeweils $8 \mu \mathrm{m}$ auf und liegen damit pulverförmig vor. Die mit Hilfe der Walzenschüsselmühle zerkleinerte Probe erzielte einen d90-Wert von $104 \mu \mathrm{m}$ und liegt somit genau im ausgewählten Körnungsbereich. Von allen zerkleinerten $\mathrm{MgO}-$ Proben wurde das Sedimentationsverhalten und der Hydratisierungsgrad bestimmt.

\subsubsection{Ermittlung des Sedimentationsverhaltens}

Die Temperaturen der Hydratisierung variieren stark in der industriellen Anwendung, daher wurden zuerst Versuche mit dem "RHIM CCM Ofenfallend" (welches direkt aus dem Drehrohrofen erhalten wird) mit einer Konzentration von $90 \mathrm{~g} / \mathrm{l}$ bei unterschiedlichen Temperaturen durchgeführt. Anschließend wurde dieser Versuch mit einer MgOKonzentration von $70 \mathrm{~g} / \mathrm{l}$ wiederholt. Die graphische Auswertung beider Versuchsserien ist in Abb. 3 und 4 dargestellt, wobei das $\mathrm{Mg}(\mathrm{OH})_{2}$-Slurry-Volumen im Verhältnis zum Startvolumen gegen die Zeit aufgetragen wurde. Zusätzlich wurde von beiden Versuchsserien im Bereich des linearen Sedimentationsverhaltens die Sedimentationsgeschwindigkeit berechnet und in Abb. 5 (Hydratisierungstemperatur gegen Sedimentationsgeschwindigkeit) graphisch dargestellt.

Ein eindeutiger Trend im Sedimentationsverhalten ist in Abb. 3 zu erkennen. Je höher die Temperatur für den Hydratisierungsprozess gewählt wurde, desto schneller lief die Sedimentation ab. Dieses Verhalten ist ebenso bei den Versuchen mit einer Konzentration von $70 \mathrm{~g} / \mathrm{l}$ beobachtbar. Nach Berechnung der erreichbaren Sedimentationsgeschwindigkeiten kann in Abb. 5 ein exponentieller Zusammenhang zwischen der Hydratisierungstemperatur und der Sedimentationsgeschwindigkeit festgestellt wer-

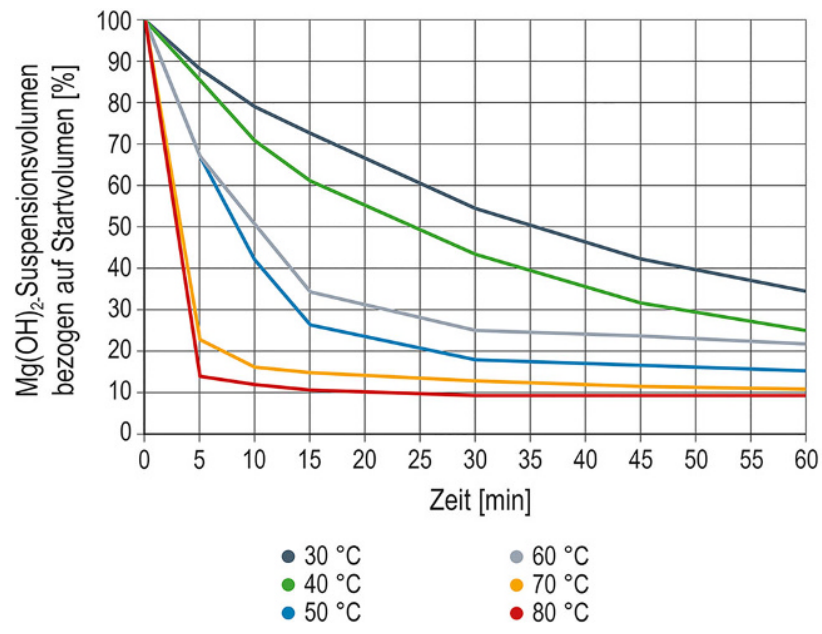

Abb. 4: Sedimentationsverhalten "RHIM CCM Ofenfallend" nach unterschiedlichen Hydratisierungstemperaturen (Hydratisierungsdauer $1 \mathrm{~h}$ ) mit einer Konzentration von $70 \mathrm{~g} / \mathrm{l}$ 


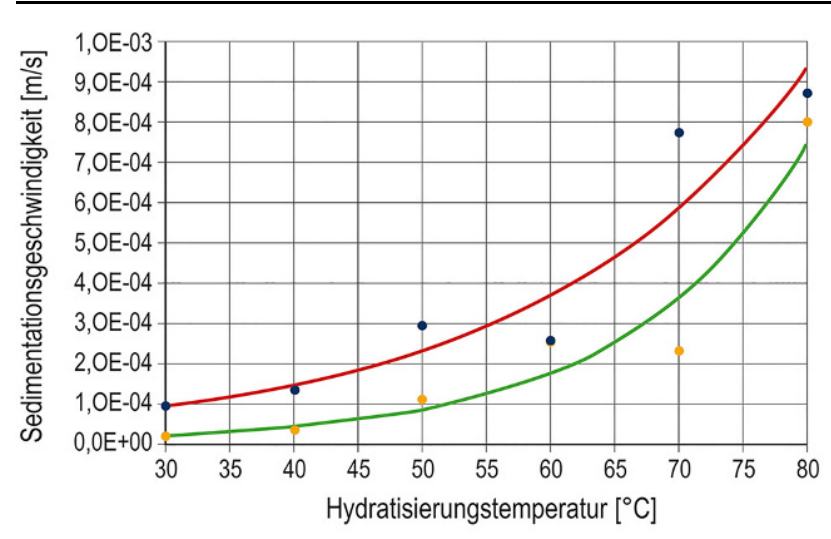

- RHIM CCM Ofenfallend 70g/l

- Exponentiell RHIM CCM Ofenfallend $70 \mathrm{~g} /$

- RHIM CCM Ofenfallend 90g/l

- Exponentiell RHIM CCM Ofenfallend 90g/l

Abb. 5: Sedimentationsgeschwindigkeiten „RHIM CCM Ofenfallend“ bei unterschiedlichen Hydratisierungstemperaturen (Hydratisierungsdauer $1 \mathrm{~h}$ ) mit einer Konzentration von 70 und $90 \mathrm{~g} / \mathrm{l}$ sowie exponentieller Trendlinie

den. Dies bedeutet, dass die Hydratisierung von MgO bei hohen Temperaturen die Sedimentationsgeschwindigkeit exponentiell erhöht. Für die Anwendung in der Papier- und Zellstoffindustrie stellt dies einen negativen Einfluss bei Anwendung von zu hohen Temperaturen im Hydratisierungsprozess dar, da hier eine möglichst langsame Sedimentation gewünscht wird.

Die weiteren zerkleinerten MgO-Proben wurden ebenfalls mit einer Konzentration von $90 \mathrm{~g} / \mathrm{l}$ bei einer Temperatur von $30^{\circ} \mathrm{C}$ hydratisiert und anschließend das Sedimentationsverhalten beobachtet. In Abb. 6 sind die Ergebnisse dargestellt und ergänzend der $d_{90}$ für jede dieser Proben angegeben. Aus dieser Graphik ist ein leichter Trend ableitbar, welcher sich wie folgt darstellt: mit zunehmender Feinheit verläuft die Sedimentation des $\mathrm{Mg}(\mathrm{OH})_{2}$ langsamer. Der "RHIM CCM Ofenfallend“ stellt dabei eine Ausnahme dar. Dieser wurde nicht aufbereitet und weist bei näherer Betrachtung Agglomerate auf, daher ein d9o von ca. $1100 \mu \mathrm{m}$. Die Agglomerate sind weich und können zum Großteil mit den Fingern zerdrückt werden, zurück bleibt ein nicht zerdrückbarer Nukleus. Bei der Hydratisierung bilden sich voluminöse $\mathrm{Mg}(\mathrm{OH})_{2}$-Partikel aus, welche sehr langsam sedimentieren. Der feste Nukleus, der innerhalb der Agglomerate zu finden ist, sinkt bei der Hydratisierung zu Boden und stellt im Speziellen in der großtechnischen Anwendung einen störenden Bodenkörper dar.

\subsection{Hydratisierungsgrad}

In Abschn. 4.2 wurden die Probenherstellung und die Auswertung zur Bestimmung des Hydratisierungsgrades beschrieben. Die erreichten Hydratisierungsgrade sowie die vorliegenden $d_{90}$-Werte und die dazu gehörigen spezifischen Oberflächen werden in Tab. 4 zusammengefasst.

Aus Tab. 4 wird ersichtlich, dass der "RHIM CCM Ofenfallend“ sowie die zerkleinerte MgO-Probe mittels Stiftmühle bei $30^{\circ} \mathrm{C}$ Hydratisierungstemperatur nach einer Stunde Hy-

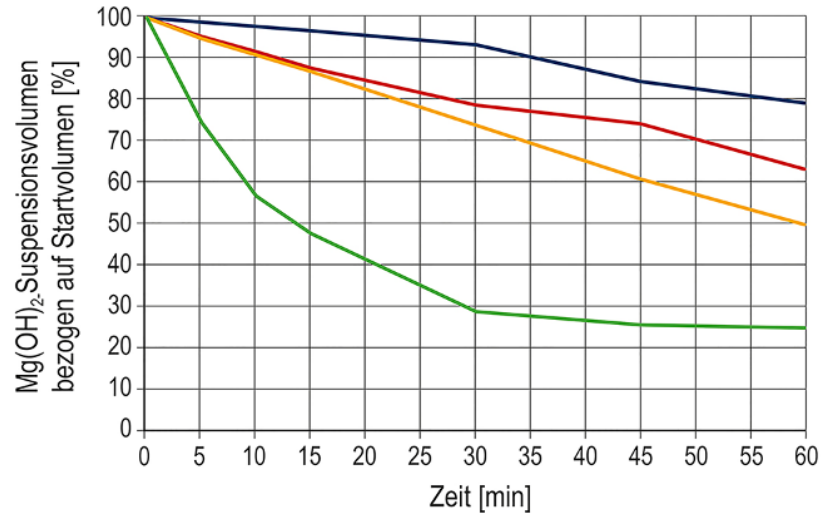

- RHIM CCM Ofenfallend $d 90=1100 \mu \mathrm{m}$

- Stiftmühle d90=10 $\mu \mathrm{m}$

- Schallstoßwellen d90=8um

- Walzenschüsselmühle d90=105um

Abb. 6: Sedimentationsverhalten „RHIMCCM Ofenfallend“ im Vergleich mit RHIM CCM aufbereitet mit Hilfe unterschiedlicher Zerkleinerungsaggregaten, Hydratisierungstemperaturen $30^{\circ} \mathrm{C}, \mathrm{Hydratisierungsdauer} 1 \mathrm{~h}$, Konzentration von $90 \mathrm{~g} / \mathrm{l}$

dratisierungsdauer einen Hydratisierungsgrad von 88 bzw. $89 \%$ erreichen. Der d 90 der beiden Proben unterscheidet sich dabei sehr stark, wobei die spezifische Oberfläche mit 38 und $39 \mathrm{~m}^{2} / \mathrm{g}$ als gleich anzusehen ist. Die mit Hilfe der Walzenschüsselmühle erhaltene $\mathrm{MgO}$-Probe weist eine etwas kleinere spezifische Oberfläche auf, der d9o beträgt hier $105 \mu \mathrm{m}$ und es wurde ein Hydratisierungsgrad von $69 \%$ erreicht. Die zerkleinerte $\mathrm{MgO}$-Probe, die den niedrigsten $\mathrm{Hy}$ dratisierungsgrad mit $52 \%$ aufweist, ist die Probe, die mittels Schallstoßwellen behandelt wurde, obwohl diese Probe die höchste spezifische Oberfläche aller $4 \mathrm{MgO}-P r o b e n$ mit $42 \mathrm{~m}^{2} / \mathrm{g}$ und einen $\mathrm{d}_{90}$ von $8 \mu \mathrm{m}$ aufweist. Aus den getesteten zerkleinerten MgO-Proben ist kein Trend in Bezug auf den zu erreichenden Hydratisierungsgrad ersichtlich. Anscheinend haben weder der Zerkleinerungsgrad noch die spezifische Oberfläche Einfluss auf diesen.

Nachdem die mittels Stiftmühle zerkleinerte MgO-Probe in den Labortests den besten Hydratisierungsgrad erreichte, ein gewünscht langsames Sedimentationsverhalten zeigte und der $d_{90}$-Wert bei rund $8 \mu \mathrm{m}$ lag, wurde in weiterer Folge diese Zerkleinerungsart für "RHIM CCM Ofen-

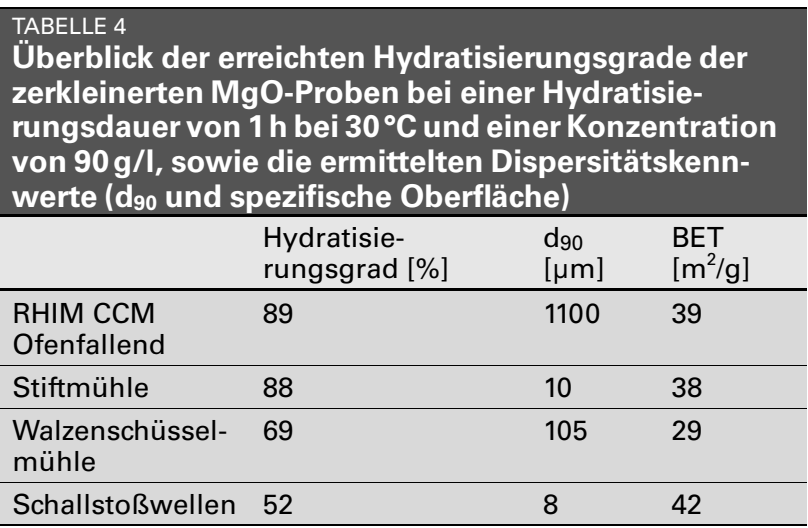




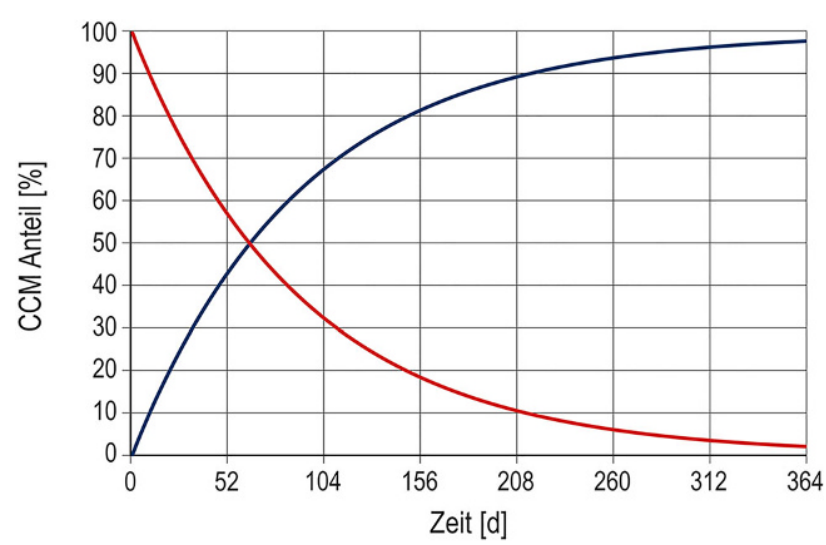

- RHIM CCM P\&P

- Standard CCM Anteil

Abb. 7: Austauschrate „RHIM CCM P\&P“ mit Standard CCM während des 4-monatigen Betriebsversuches, Rezyklierungsrate MgO von $94 \%$

fallend“ ausgewählt, um damit das Produkt "RHIM CCM P\&P“ herzustellen.

\section{Ergebnisse - Einsatz im industriellen Maßstab}

In einer Zellstofffabrik eines namhaften Papier- und Zellstoff-Herstellers wurde Mitte letzten Jahres das Produkt „RHIM CCM P\&P“ über einen Zeitraum von vier Monaten sortenrein in dessen Anlage getestet. Dabei wurde besonderes Augenmerk auf die Betriebstemperatur während der Hydratisierung des $\mathrm{MgO}$ und deren Konzentration in der herzustellenden $\mathrm{Mg}(\mathrm{OH})_{2}$-Suspension gelegt. Weiters wurde die Absorption des $\mathrm{SO}_{2}$ aus dem Rauchgas beobachtet.

Der Prozess der Rauchgasentschwefelung wird in dieser Anlage nach dem Babcock-Verfahren durchgeführt. Das $\mathrm{MgO}$ wird zu ca. $94 \%$ rezykliert und die Verluste von $6 \% \mathrm{mit}$ sog. Make-Up MgO ausgeglichen. Nach einer Betriebsdauer von vier Monaten konnte eine Austauschrate von knapp $75 \%$ erreicht werden (siehe Abb. 7). Um einen 100-prozentigen Austausch von bisher verwendetem Standardmaterial durch "RHIM CCM P\&P“ zu erreichen, müsste eine Einsatzdauer von mindestens einem Jahr erfolgen.

Für den Betriebsversuch wurden rund $400 \mathrm{t}$ "RHIM CCM Ofenfallend“ mittels Stiftmühle aufbereitet, per Silo-LKW zur Zellstofffabrik angeliefert und anschließend eingesetzt. Während des Versuches konnte die Hydratisierungstemperatur bei der Umwandlung von $\mathrm{MgO}$ zu $\mathrm{Mg}(\mathrm{OH})_{2}$ von 90 auf ca. $70^{\circ} \mathrm{C}$ reduziert werden. Dadurch konnten $64 \%$ des zur Einstellung der Prozesstemperatur benötigten 3-bar Heißdampfes eingespart werden. Die MgO-Konzentration bei der Herstellung der $\mathrm{Mg}(\mathrm{OH})_{2}$-Suspension wurde aus betrieblichen Gründen nicht reduziert. In einem nächsten Versuch soll auch dieser Parameter schrittweise optimiert werden, um den MgO-Einsatz bis zur möglichen Minimalkonzentration zu senken.

Die Absorption des $\mathrm{SO}_{2}$ aus dem Rauchgas mit Hilfe der im ersten Schritt produzierten $\mathrm{Mg}(\mathrm{OH})_{2}$-Suspension konnte problemlos in der dafür verwendeten Venturikaskade

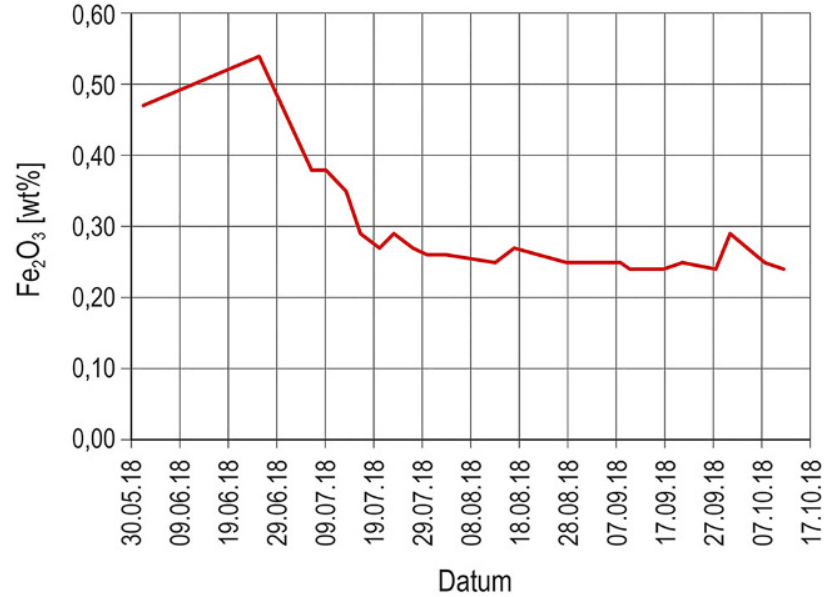

Abb. 8: Verlauf $\mathrm{Fe}_{2} \mathrm{O}_{3}-\mathrm{Gehaltes}$ während des 4-monatigen Betriebsversuches im rezyklierten $\mathrm{MgO}$

durchgeführt werden. Durch den hohen Hydratisierungsgrad von fast $90 \%$ konnte wesentlich mehr $\mathrm{SO}_{2}$ absorbiert werden, wodurch die Aufstärkung der sogenannten Rohsäure mit Flüssig- $\mathrm{SO}_{2}$ ebenfalls reduziert werden konnte. Die Reduktion betrug während des Betriebsversuches an die $24 \%$.

Die Einbringung von Störstoffen konnte merklich reduziert werden, wie am Beispiel von Eisen(III)-oxid $\left(\mathrm{Fe}_{2} \mathrm{O}_{3}\right)$ in Abb. 8 gezeigt wird. Hier ist ab dem Zeitpunkt des Einsatzes von „RHIM CCM P\&P“ eine Absenkung des $\mathrm{Fe}_{2} \mathrm{O}_{3}$-Gehaltes um $50 \%$, von anfangs 0,5 auf $0,25 \%$, zu erkennen, welcher im rezyklierten $\mathrm{MgO}$ direkt nach der Abscheidung im Elektrofilter mittels RFA bestimmt wurde.

Nach viermonatigem Einsatz von "RHIM CCM P\&P“ konnten schon kleine Einsparungen und positive Effekte auf den gesamten Rauchgasentschwefelungs- und dessen Folgeprozess festgestellt werden. Das gesamte Einsparungspotential von "RHIM CCM P\&P“ ist aber noch viel höher einzuschätzen, da das Produkt erst zu $75 \%$ und nicht zu 100\% im Produktionskreislauf vorhanden war. Weiteres Optimierungspotential wird in der Absenkung der Hydratisierungstemperatur sowie in der Reduktion des spezifischen MgO-Bedarfes gesehen, dadurch ergeben sich weitere Energie- und MgO-Einsparungen. Durch die minimierte Einbringung an Störstoffen können in weiterer Folge Instandhaltungskosten gesenkt werden, da Ablagerungen und Verschleiß reduziert werden. Ein zusätzlicher positiver Effekt der geringen Störstoffkonzentration von

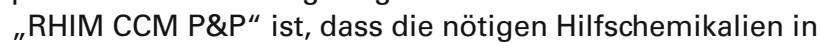
den Folgeprozessen (wie z. B. Bleichchemikalien) ebenso in ihrem Einsatz minimiert und damit die Produktionskosten weiter gesenkt werden können.

\section{Zusammenfassung}

Es konnte aufgezeigt werden, dass die Hydratisierungstemperatur bei der Umsetzung von $\mathrm{MgO}$ zu $\mathrm{Mg}(\mathrm{OH})_{2}$ einen Einfluss auf das Sedimentationsverhalten hat. Je höher die Temperatur gewählt wird, umso schneller läuft die 
Sedimentation ab. Nach Berechnung der Sedimentationsgeschwindigkeiten für die Suspensionen mit 70 und $90 \mathrm{~g} / \mathrm{l}$ von „RHIM CCM Ofenfallend“ konnte ein exponentieller Zusammenhang zwischen Hydratisierungstemperatur und Sedimentationsgeschwindigkeit festgestellt werden. Optimal für die Anwendung in der Papier- und Zellstoffindustrie ist somit eine Temperatur von $30-50^{\circ} \mathrm{C}$, da in diesem Bereich die Sedimentation des $\mathrm{Mg}(\mathrm{OH})_{2}$ langsam abläuft. Ein eindeutiger Zusammenhang zwischen zerkleinerungstechnischen Parametern und dem Sedimentationsverhalten bzw. dem zu erreichenden Hydratisierungsgrad konnte bis dato nicht erkannt werden. Die besten Laborergebnisse wurden mit der durch die Stiftmühle zerkleinerten MgOProbe erzielt. Daher wurde das Produkt "RHIM CCM P\&P“ durch Zerkleinerung von "RHIM CCM Ofenfallend“ mittels Stiftmühle für einen großindustriellen Einsatz produziert. Dieser 4-monatige Betriebseinsatz von "RHIM CCM P\&P“ kann positiv bewertet werden, da die Anforderungen der Papier- und Zellstoffindustrie erfüllt wurden. Schon nach kurzer Einsatzdauer konnten positive Effekte im gesamten Rauchgasentschwefelungsprozess sowie in den Folgeprozessen erzielt werden.

Funding. Open access funding provided by Montanuniversität Leoben.

Open Access Dieser Artikel wird unter der Creative Commons Namensnennung 4.0 International Lizenz (http://creativecommons.org/licenses/ by/4.0/deed.de) veröffentlicht, welche die Nutzung, Vervielfältigung,
Bearbeitung, Verbreitung und Wiedergabe in jeglichem Medium und Format erlaubt, sofern Sie den/die ursprünglichen Autor(en) und die Quelleordnungsgemäßnennen, einen LinkzurCreativeCommons Lizenz beifügen und angeben, ob Änderungen vorgenommen wurden.

\section{Literatur}

1. Blechschmidt, J. (Hrsg.): Taschenbuch der Papiertechnik, 2. Auflage, München: Fachbuchverlag Leipzig im Carl Hanser Verlag, 2013

2. CEPI-Confederation of European Paper Industries: The Forest Fibre Industry-2050 Roadmap to a low-carbon bio-economy, (C) 2011 CEPI 3. Schloffer, K.: Sulfitzellstoffherstellung Teil 1, Schulungsunterlagen 2018, (c) Added Value International

4. Gullichsen, J.; Paulapuro, H.(eds.): Papermaking Science and Technology-Book 6A, Chemical Pulping, Helsinki: Finnish Paper Engineers' Association and TAPPI, 1999

5. Shand, M. A.: The Chemistry and Technology of Magnesia, Hoboken, NJ: Wiley Interscience, 2006

6. Birchal, V.S.; Rocha, S.D.F.; Mansur, M.B.; Ciminelli, V.S.T.: A Simplified Mechanistic Analysis of the Hydration of Magnesia, The Canadian Journal of Chemical Engineering, Vol 79 (2001), iss. 4, pp 507-511

7. Salomão, R.; Arruda, C.C.; Kawamura, M.A.: A systemic investigation on the hydroxylation behavior of caustic magnesia and magnesia sinter, Ceramics International, Vol 41 (2015), iss. 10, Pt. B, pp 13998-14007

Hinweis des Verlags. Der Verlag bleibt in Hinblick auf geografische Zuordnungen und Gebietsbezeichnungen in veröffentlichten Karten und Institutsadressen neutral. 